\title{
A Network-Type Brain Machine Interface to Support Activities of Daily Living
}

Takayuki SUYAMA ${ }^{\dagger a)}$, Member

\begin{abstract}
SUMMARY To help elderly and physically disabled people to become self-reliant in daily life such as at home or a health clinic, we have developed a network-type brain machine interface (BMI) system called "network BMI" to control real-world actuators like wheelchairs based on human intention measured by a portable brain measurement system. In this paper, we introduce the technologies for achieving the network BMI system to support activities of daily living.

key words: brain machine interface, smart house, data analysis, network agent
\end{abstract}

\section{Introduction}

Brain machine interface (BMI) technology that connects human brain information to a machine with the aim of manipulating the latter is expected to help physically disabled people and to the elderly live independent lives. There have been many studies of BMI systems to date [1]-[5], but most of these have been performed as laboratory experiments. To bring BMI systems to a more practical level, they need to be researched and tested not just in the laboratory but also at locations that approximate actual living conditions. However, such real-world environments are not as clean as a laboratory and they require support for scenarios not considered in a laboratory such as indoor mobility while wearing a BMI. At the same time, there has also been research on smart houses that install indoor sensors or other devices [6]-[8]. These studies also feature the simultaneous acquisition of brain information. The system for achieving BMI in the daily living environment consists of a combination of platform technologies; i.e. portable brain-information sensing technology, network technology based on software agents [9], real-world experimental facilities, brain-information decoding technology [10]-[14] and device control technology based on brain information [15]. This paper summarizes these technologies in the following sections.

\section{Network BMI}

Network BMI is outlined in Fig. 1. Reflecting the central theme of this research, we first constructed a "BMI house" as an experimental environment in which brain information and environmental information could be simultaneously obtained. In this environment, the system measures user

\footnotetext{
Manuscript received May 30, 2016.

$\dagger$ The author is with ATR Cognitive Mechanisms Laboratories, Kyoto-fu, 619-0288 Japan.

a)E-mail: suyama@atr.jp

DOI: $10.1587 /$ transcom.2016SNI0002
}

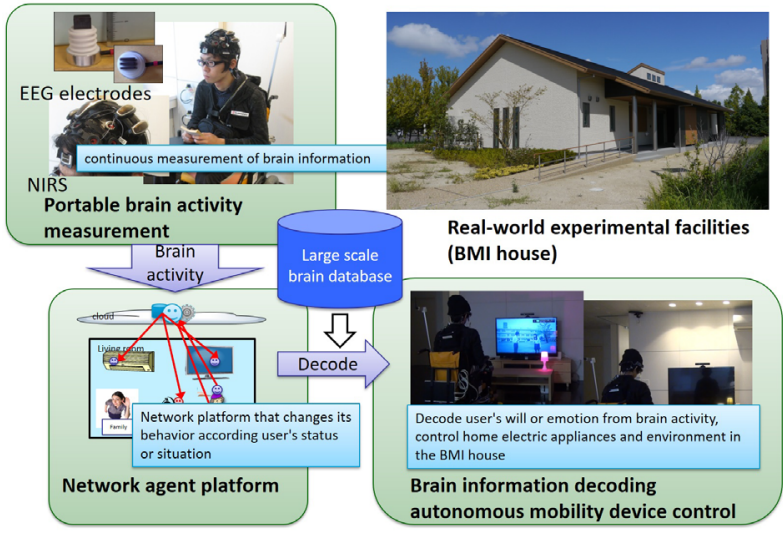

Fig. 1 The outline of the network BMI system.

brain information using portable brain-activity measurement equipment and transfers that information using network platform technology. It then analyzes the information using brain-information analysis technology together with a largescale brain-activity database and uses the results of that analysis to control a wheelchair, home appliances, etc. The following describes each of these research topics in detail.

\section{High-Accuracy Brain-Information Sensing Technol- ogy}

This section describes technology for performing noninvasive, high-accuracy, and continuous measurements of brain information using portable equipment. There are two types of brain-information measurement methods that can be used with portable equipment: electroencephalography (EEG) for measuring brain waves and near-infrared spectroscopy (NIRS) for measuring changes in the concentration of oxygenated hemoglobin and deoxygenated hemoglobin in brain blood flow. In the research presented here, we developed semi-dry electrodes and dry electrodes (Fig. 2) to measure brain waves by EEG with high accuracy in a real-world environment. The semi-dry electrodes are of the silver-silver chloride type having low polarization potential. They feature a brush-like structure that parts the hair while having a polishing effect on the scalp. They also use a polymer gel sheet containing water molecules for the electrode substrate to ensure long-term stability covering a period of at least four hours. The dry electrodes, meanwhile, consist of three parallel plates as a spring-supported blade structure based on the way that hair tends to orientate. Targeting both of 


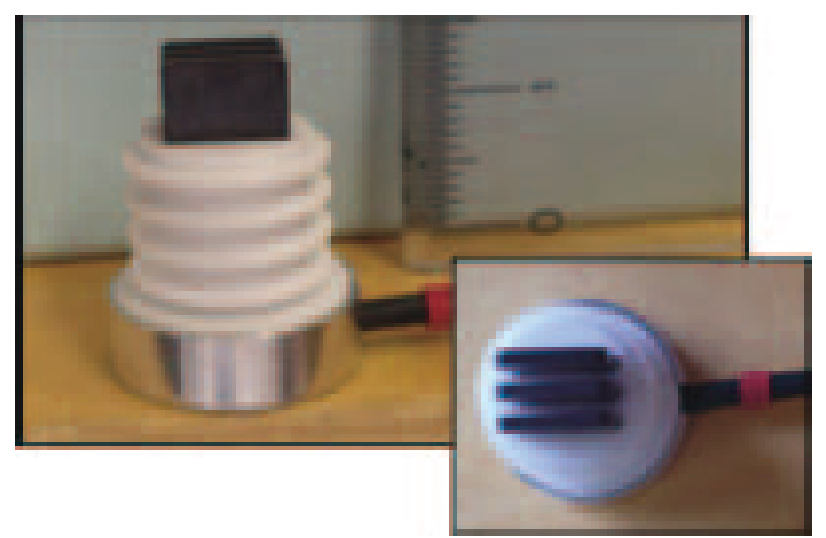

Fig. 2 Dry EEG electrodes.

these types of electrodes, we performed an experiment to see whether they could be used to perform continuous recording for at least four hours while being appropriately mounted on the user's head. Based on the results obtained, we prepared an environment in which either type of electrode could be selected depending on the application. Specifically, we would use semi-dry electrodes for experiments with much head fluctuation and long continuous recording times and dry electrodes for experiments with little head fluctuation and with the need to shorten experimental preparations.

We also developed network-BMI brain-activity measuring equipment consisting of portable brain-activity measuring equipment, external brain-activity measurement and control equipment, and external brain-activity data observation equipment. For the portable brain-activity measuring equipment, we developed technology for everyday acquisition of brain-activity data in the form of NIRS-EEG probes and a NIRS-EEG unit that enables continuous and simultaneous measurements of brain activity by NIRS and EEG. The NIRS-EEG probes consist of 8 channels of NIRS probes and 10 channels of EEG electrodes that can be adjusted to simplify head mounting and accommodate individual differences. The NIRS-EEG unit, meanwhile, can be converted into various types depending on the experimental format, such as a wheelchair-mounted type for powered wheelchair experiments and a body-bag type for experiments involving simultaneous measurements by multiple units of portable brain-activity measuring equipment involving multiple users. We eventually settled on a "bag-pack" type that could be used both as a personal unit and a wheelchairmounted unit. Next, to achieve a function for transmitting acquired data, we used a $5 \mathrm{GHz}$ wireless LAN system to avoid interference with the $2.4 \mathrm{GHz}$ band commonly used by smartphones and home appliances capable of wireless communications. In this way, we achieved stable, simultaneous measurement by multiple units (four) of portable brain-activity measuring equipment for a prolonged period of time ( 3 hours or more, 24 hours of data in total). In addition, the external brain-activity measurement and control equipment can set parameters, perform calibration, and control measurements for multiple units of portable brain-

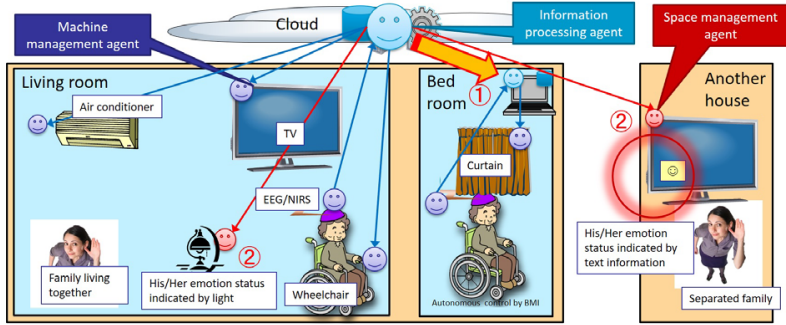

Fig.3 Network agent platform.

activity measuring equipment simultaneously. Finally, the external brain-activity data observation equipment can observe measured data, data measurement conditions, and data processing conditions for multiple units of portable brainactivity measuring equipment simultaneously.

\section{Network Technology for Integrating Brain Informa- tion and Real-World Information}

In this research, we developed a network agent platform based on software agents as a technology for analyzing brain information on a cloud in the network (Fig. 3). First, with the aim of conducting a basic study on the architecture needed for a distributed management and processing platform targeting brain-activity data and real-world information and a study on search technology for low-delay decoding, we adopted a modeling technique using software components (referred to below as "agents") that treat data and processing on equal terms. We then used this agent model as a basis for investigating distributed management techniques for brainactivity data, distributed processing techniques for brainactivity decoding, and distributed management techniques for real-world information. Next, we designed basic architecture based on a new model that extends an existing model dividing real space into three types of agents corresponding to people, devices, and space. Finally, we constructed a prototype evaluation environment based on this architecture to assess the validity of the architecture's basic design and to evaluate and compare techniques. Next, we achieved a demonstration system for controlling actual equipment such as home appliances and mobile-support devices and confirmed that BMI control in a multi-user environment could be processed with a delay time less than $1 \mathrm{~s}$ for each user. Additionally, in terms of extendibility, we found that brainactivity data for several tens of persons could be processed with an equivalent delay time. Moreover, we constructed a demonstration system to test an emotional-state communication support technique using brain information in daily living and found that processing for a service using that technique could be performed with a 2-3 s delay.

\section{Construction of Real-World Experimental Facilities}

In this research, we constructed a real-world environment called "BMI house" (Figs. 4 and 5) for conducting experiments under actual living conditions. Similar to ordinary 


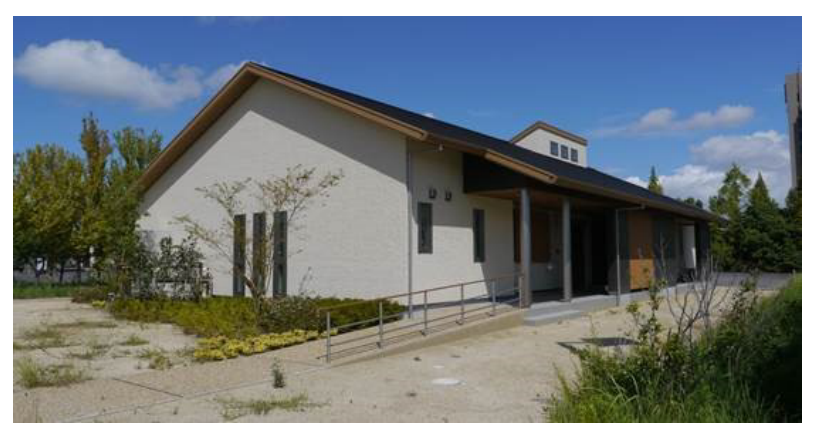

Fig. 4 BMI house.

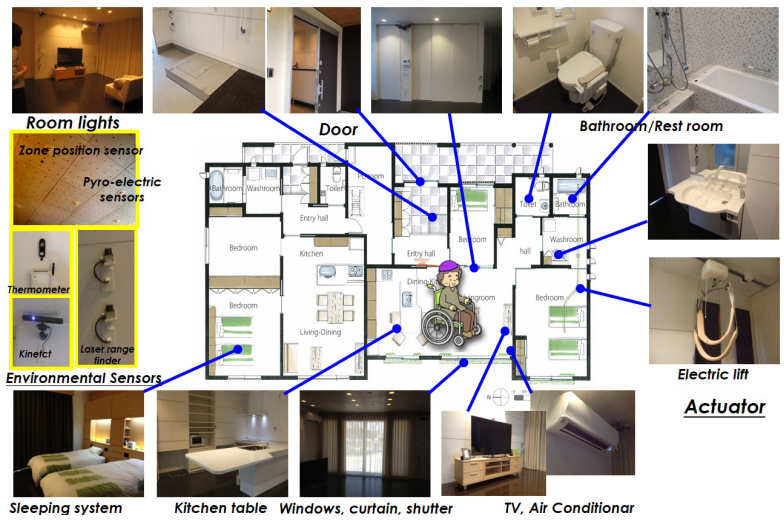

Fig. 5 Interior of BMI house.

smart homes, this experimental house installs many sensors plus the ability to simultaneously measure brain information. These sensors include cameras, microphones, and infrared devices for detecting the position and status of people and measuring the indoor environment. Transmission technology for connecting sensor-obtained data to a data processing computer on the network is installed in the BMI house. We also designed remotely operable residential facilities, home appliances, etc. as actuators supporting living and caregiving in daily living space and installed them in the BMI house. These facilities and home appliances could be externally operated by a control system. We also evaluated an optimal environment control function and an actuator operation control function achieved by combining brain information with other biological and environmental information, and assessed the possibility of achieving a practical living space using network BMI. In addition, we evaluated and assessed the feasibility of a sleep support system that uses brain activity and biological data to adjust the indoor environment via air conditioning, lighting, etc. Moreover, we constructed a prototype living support system applying an emotional-state communication support service using brain information in an ordinary living environment and assessed its usefulness. Additionally, taking into account long-term daily living experiments, we determined a sensor arrangement for detecting the position and status of people and measuring the indoor environment using cameras, microphones, infrared devices, etc. and formulated actuator specifications for various types of devices and appliances used in daily life. On completing the construction of these real-world experimental facilities, we checked their operation by real-equipment demonstrations and other means.

\section{Brain Activity Decoding}

In this section, we describe the technologies for brain activity decoding. Firstly, we constructed a brain activity database to realize the data-driven brain decoding algorithm. Secondly, we introduce several brain decode algorithms. Finally, we describe the experiment of the BMI based on NIRS measurement.

\subsection{Construction of Brain-Activity Database}

In this research, we constructed a brain-activity database for analyzing brain information in a data-driven manner. The "tagged brain-activity databases" that we obtained in this research includes a multi-person/short-term reference brainactivity database (on a level of 100 persons each measured for about one hour) as an aid in constructing a BMI decoding algorithm. First, with the aim of establishing BMI decoding technology based on natural brain activity, we obtained the participation of 62 experimental subjects and constructed a reference brain-activity database consisting of multiple measurement modes (such as large-scale brain measurement equipment as in fMRI and portable brain measurement equipment as in EEG-NIRS) using spatial attention tasks. Next, to establish BMI decoding technology that can be used for emotional-state communication support, we constructed a reference brain-activity database also consisting of multiple measurement modes (fMRI, EEG, behavioral experiments, psychological indices, etc.) using tasks that present images evoking pleasant and unpleasant emotions. As for the latter, we excluded subjects participating in pilot experiments and those who could not participate in either EEG or fMRI measurements, and as a result, we obtained data for more than 50 subjects in both EEG and fMRI modes. Finally, we combined the above databases to construct a multi-person (more than 100 persons), short-term reference brain-activity database covering both large-scale brain measurement equipment and portable brain measurement equipment.

Next, our "tagged brain-activity databases" also include a multi-person/long-term (more than 24 hours in total) tagged brain-log database obtained under daily living conditions. First, using two units of early-type portable brain-activity measurement equipment and the participation of two experimental subjects, we measured brain activity, biological conditions, and the indoor environment while living in the BMI house for a total of 26.5 hours of measurements. We then integrated the results obtained and added information such as behavior labels to construct a tagged brain-log database. Next, we developed an enhanced version of portable brainactivity measurement equipment by increasing the number of EEG channels, improving the hardware of the measurement equipment, improving the wireless communications system, 
etc. This gave us a maximum of four units of portable brain-activity measurement equipment including the earlytype units. Using these units, we conducted a long-term measurement experiment lasting five days using three experimental subjects participating in a variety of actions and scenarios typical of daily life. In this experiment too, we measured brain activity, biological conditions, and the indoor environment. Then, by integrating these measurement results and adding information such as behavior labels, we constructed a tagged brain-log database having more than 30 hours of data in total.

Here, to make the manual attachment of behavior labels to the data obtained in these long-term measurement experiments more efficient, we developed a labeling support tool that incorporates a function for automatically extracting actions performed by an experimental subject from images obtained using multiple environmental cameras. We have also been researching semi-automatic behavior labeling technology that will become necessary when obtaining more experimental data from long-term measurements in the future. In this research, we have so far developed several types of behavior recognition systems and evaluated their performance. These systems use different types of sensor information including skeleton information from a gesture sensor (Kinect), environmental camera images, wearable motion-sensor information, and images from head-mounted cameras.

\subsection{Real-Time Brain-Information Extracting/Decoding Technology}

We researched and developed a data-driven brain decoding method. This is a method that collects a large volume of data and analyzes brain information using machine learning techniques. Taking into account the need for standardization in the coding/decoding of brain information, we have been researching and developing robust feature extraction methods that can be applied to real-world environments based on the common spatial pattern (CSP) procedure used as standard in methods for decoding brain information from brain waves. At the same time, we have come to design a data-driven brain-information decoding algorithm by referencing standard technology used in multimedia searching and retrieval. First, we constructed a prototype data-driven brain-information decoder using the reference brain-activity database created from EEG-BMI experimental data assuming movement in the BMI house and conducted a BMI control experiment targeting a wheelchair and home appliances. In this experiment, it was found that (two) experimental subjects leading a wheelchair life in the BMI house could operate mobility support devices and home appliances by BMI with a decoding delay under $1 \mathrm{~s}$ (where delay is measured from the initial sending of brain-information features to the receiving of control signals at the target device). Average accuracy at this time was found to be $77.7 \%$. Furthermore, when setting conditions such that system delay time (total of round-trip communication time and decoding time) would be under $1 \mathrm{~s}$, average accuracy was found to be $82.7 \%$ after adding two people to the two above for a total of four experimental subjects.

We next developed a network BMI system supporting multiple users. Using this system, we performed an evaluation test by having two experimental subjects wear different portable EEG units in the BMI house and having them repeatedly operate living support devices by BMI assuming movement. A delay time less than $1 \mathrm{~s}$ was achieved for both of these subjects. Additionally, we also conducted a demonstration experiment of mobility control for multiple mobility support devices based on this network BMI system at a health clinic (Sincere Korien, Day Care Center). In this way, we were able to present the eventual image of integrating network BMI with safe-and-secure autonomous mobility support technology in an actual usage scenario.

Furthermore, on analyzing the tagged brain-log database, we discovered that natural brain activity corresponding to an operation-type intention associated with movement could be identified with a single NIRS measurement signal. We therefore developed a new operationidentification-type BMI based on NIRS measurement making use of this principle. We applied this technique to identifying three types of operations (turning on a TV, turning on an air conditioner, and reading a book) achieving an accuracy of $84 \%$. We describe this experiment in next section in detail.

We next describe our research of emotional-state communication support technology. Our aim here was to differentiate between a tense state and relaxed state, which are two important emotional states in daily life. To this end, we performed EEG measurements under "tense conditions" (when imagining some occurrence that generated anxiety or stress while listening to music that induces tension) and "relaxed conditions" (when imagining a relaxed situation while listening to music that induces relaxation). On using this data to evaluate system performance in discriminating between these two conditions, two out of three experimental subjects achieved an accuracy rate greater than 70\%. In addition, using a brain-information decoder capable of discriminating between a tense state and relaxed state, we constructed a network BMI system that actuates (visualizes) an "anxious/tense emotional state" as the color of a ball lamp. We then showed as a usage scenario how this system could be used as a tool for conveying to caregivers or helpers the emotional state of the person being cared for. Furthermore, when using the system online, we found average delay time to be $0.46 \mathrm{~s}$ and total delay time including measurement time by portable brain-activity measuring equipment to be within $2.0-2.7 \mathrm{~s}$

We next describe our research of real-space actuator control by network BMI. With the aim of supporting persons with upper-limb disabilities in need of care, we developed a wearable mobility support actuator. Moreover, to support daily tasks in the BMI house by brain information decoding, we implemented a link between this wearable mobility support actuator and home devices and integrated this link with brain information, myoelectric information, and joint-angle 


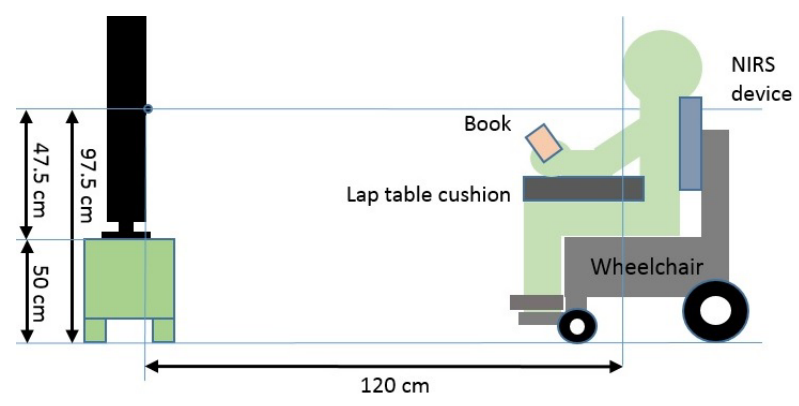

Fig. 6 Experimental setting of BMI based on NIRS.

information. Then, as a use case for the wearable mobility support actuator, we constructed a system to demonstrate support for the following sequence of tasks: moving and picking up a cup, moving again while holding the cup, holding out the cup under a faucet and filling it with water, and bringing the cup up to one's face to drink the water.

\subsection{BMI Based on NIRS Measurement}

In this section, we describe the experiment results of NIRS based BMI [14]. In this experiment, The participant sat on a wheelchair at a distance of $1.2 \mathrm{~m}$ from the television, holding a book with both hands on a cushioned lap desk in a relaxed manner as shown in Fig. 6. A single task session includes a number of repeated trials of four daily-living actions (DLAs), shown in Fig. 7. In each trial, first, a number (1 to 4) was displayed for one second, initiated with a short beep sound. Then, a white cross fixation appeared on the TV, which cued the participants to start performing the instructed DLA slowly and to keep the posture for five seconds. Finally, the participant returned their arms to the initial position after the white cross fixation disappeared. Inter-trial interval was 30 seconds, which was long enough to reset the NIRS signal to baseline. A single session consisted of 40 trials in total (10 trials for each DLA), and took about 20 minutes.

To carry out this experiment, we enrolled three male adults (participants \#01 - \#03). They are all right-handed, aged between 24 and 42, in good health, with normal or corrected to normal vision, and with no history of neurological disorders or medication use. They performed two to four sessions (per day) in each condition of the DLA task over ten, three, and two days, respectively; the total numbers of task trials (sessions) per condition were 800 (20), 240 (6), and $160(4)$, respectively.

We conducted the DLA task in two different conditions: (i) DLA without head movement, (ii) DLA with natural head movement. In condition (i), the participant made efforts to minimize their head movement during DLA in the recording period. In condition (ii), the participant performed DLAs without any restriction to their head movements as in natural daily-living situations.

We describe the features and class labels to process the NIRS data. We used a sliding window method to obtain multiple feature vectors from each trial of the preprocessed
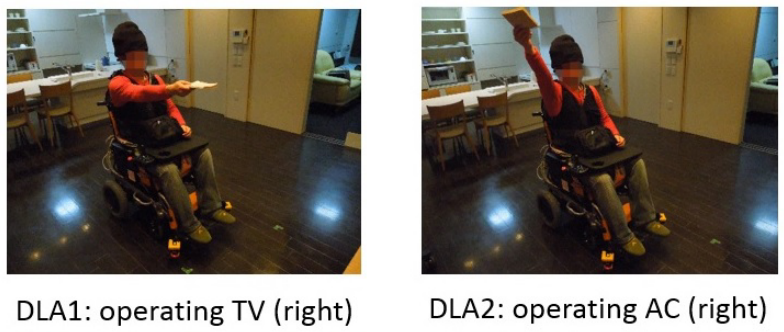

DLA2: operating AC (right)

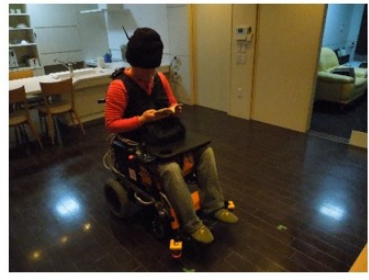

DLA3: reading a book (left and right)

Fig. 7 Four daily-living actions (DLAs).

NIRS signals. We set the window width as about 5 seconds (66 samples) and shifted it along the time axis to obtain 18 windows per trial between about 4 and 13 seconds after the stimulus onset, with the slide width of about 0.25 second. Each window was further processed to give a feature vector as follows. We first down-sampled the signals in the window by picking up one from every six samples, and then concatenated the 16-dimensional signal vectors at 11 time points into a single vector having $16 \times 11=176$ elements. Finally, we standardized the mean and variance (i.e., z-scored) the 176 elements to obtain the feature vector. Each feature vector was paired with one of the four class labels (DLA1, 2, 3 and 4) according to the DLA performed in the corresponding trial.

In order to classify each feature vector into one of the four classes of DLAs, we used a linear support vector machine (SVM) [16]. The final output for each trial was obtained by majority vote based on the 18 classification results per trial. We evaluated the offline classification accuracy in each of the two conditions (i) and (ii), using a "leave-onesession-out" type of cross-validation.

We evaluated the classification performance in each of the two conditions (i) and (ii). The result is shown in Fig. 8. In condition (i), the mean classification accuracy was about $70 \%$ for every participant, and was significantly higher than the chance level of $25 \%$. In condition (ii), the mean accuracies were lower than those in condition (i), however the participants \#01 and \#02 still exhibited significantly higher accuracies than the chance level. In principle, NIRS signals may arise from not only brain activities but also body (in particular, head) movements [17]. The higher classification accuracy in condition (i) suggests that the movement-related fluctuations in NIRS signals, which are unavoidable in natural situations, actually contained discriminative information about motions; yet the accuracy in condition (ii), significantly above the chance level, implies that the brain activity 


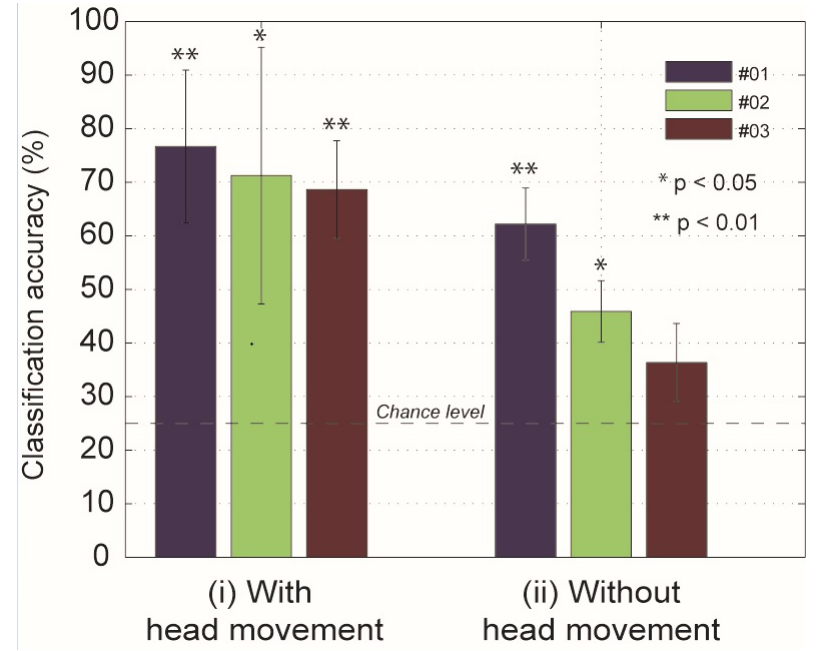

Fig. 8 Experimental result of BMI based on NIRS.

measured in the NIRS signals also had a solid contribution to the classification of DLAs.

\section{Actuation Devices Based on Brain Information}

In this section, we describe the device actuation technologies based on brain information. Since it is difficult to control devices continuously, we developed the technologies to control devices autonomously and safety.

\subsection{Living-Support Device Control Technology Based on Brain-Information Decoding}

Since continuous control of a mobility support device like a wheelchair by BMI is difficult, it is preferable to have the wheelchair move autonomously and safely after deciding on its destination by BMI or other means. In this research, we achieved an autonomous mobility function for use in mobility support devices. That is, by using only sensing functions installed on the mobility support device itself and assuming indoor paths with good visibility, we achieved safe movement that avoids collisions without the assistance of a caregiver for three types of mobility support devices (standard wheelchair, omnidirectional moving wheelchair, autonomous moving bed). In more detail, we installed sensors and a PC interface on a powered wheelchair and powered moving bed as mobility support devices, developed and installed basic operation control software and obstacle avoidance software having the functions of obstacle detection and avoidance, self-localization, mobility control, emergency stop, and safety stop, and conducted an operation evaluation experiment for these two types of mobility support devices. Results revealed that collisions could be avoided in an environment with good visibility. First, we achieved a function for avoiding entry into a danger zone that cannot be detected by sensors. This involved the construction of a grid map indicating danger zones and the implementation of software for editing map information. We also proposed a danger-zone avoidance algorithm using the grid map, installed it in a mobility support device, and confirmed that danger zones could be avoided. Next, given a command input delay (the time for a command to be returned to a mobility support device after brain activity of the mobilitysupport-device rider has been measured and transmitted) of $500 \mathrm{~ms}$, we proposed a function for generating a mobile path for safe movement. We then installed it on a mobility support device and confirmed that paths generated in accordance with this delay were safe posing no danger such as collisions. Moreover, by integrating individually developed systems and using only sensing functions installed on the mobility support device itself, we achieved a function to enable a mobility support device to avoid collisions and move safely on indoor paths with good visibility without the assistance of caregivers or helpers. In short, when using any one of the three types of mobility support devices described above (standard wheelchair, omnidirectional moving wheelchair, autonomous moving bed) and assuming conditions in which the user does not encounter or pass by other persons, we confirmed that the user could arrive at the desired destination using BMI and biological information.

\subsection{Mobility Support Device and Control System for Stress-Free Mobility}

We achieved a control system for moving a mobility support device by an autonomous mobility function as described above. This system considers the speed of the mobility support device, distance from walls, and field of view of the mobility-support-device rider (all for checking whether movement is sticking to the path, is not heading into any walls, etc.). An experiment performed on a scale of several tens of experimental subjects revealed that more than $70 \%$ felt no fear when using a mobility support device controlled by this system. In more detail, we performed an experiment to survey healthy individuals on what constitutes a stressfree and comfortable ride from the viewpoint of a mobilitysupport-device rider. In this way, we clarified three study items as conditions for stress-free and comfortable movement: the next situation can be anticipated evoking a sense of ease or danger, the next situation cannot be anticipated evoking anxiety, and the quality of the ride itself. Next, we conducted an experiment to quantify the relationship between sense of ease in the mobility-support-device rider and mobility parameters (speed and distance from walls). Based on the results obtained, we installed a prototype mobile-path planning algorithm taking sense of ease in the rider into account. In an experiment comparing this algorithm with a shortest-path mobility-planning algorithm, 26 out of 29 experimental subjects returned replies stating that they felt a greater sense of ease with the mobile-path planning algorithm that considers the rider's sense of ease. Furthermore, to model changes in sense of ease according to differences in riders' fields of view, we quantified the quality of a rider's visibility with respect to the direction of motion of the mobility support device using a 3D environmental map and 
introduced a visibility index. We then proposed a mobilepath planning algorithm that minimizes the length of the mobile locus based on this index. Finally, we integrated an evaluation index based on a map depicting distances to objects and stress-free speeds on paths with good visibility and an evaluation index rating visibility at corners and proposed a technique for use in path planning. On having 30 experimental subjects experience paths generated by the proposed technique, 22 of them or $73 \%$ felt no fear on those paths. This means that we achieved our final objective of having $70 \%$ or more users feel no fear.

We also developed mobility-support-device safety control technology linked with environmental sensors. In this development, for somewhat complicated indoor environments such as health clinics having corners, we proposed and implemented a technique that checks for people moving within the range of $m$ peripheral areas surrounding the mobility support device (including areas not seen by sensing functions installed on the mobility support device) and that checks the position and attributes of other mobility support devices and entities (people, wheelchairs, walking aids, and carts). In an actual health clinic in which an experiment could be performed, it was found that this technology could achieve attribute recognition accuracy greater than $81 \%$ with an average of $90.2 \%$. In more detail, we first developed a technique for recognizing four types of attributes (people, wheelchairs, walking aids, and carts) using laser range sensors and 3D range sensors as technology for recognizing the attributes of moving objects by environmental sensors. In an actual health clinic (Sincere Korien), we installed such environmental sensors and conducted a measurement experiment that demonstrated the effectiveness of this attribute recognition technique. Next, we developed a technique for recognizing attributes based on shape using data from $3 \mathrm{D}$ range sensors measured and accumulated at the above health clinic for those four types of attributes. We found that recognition accuracy greater than $88 \%$ could be achieved for all attributes in an environment with good visibility using feature quantities focused on measured height and target position. In addition, by enhancing this technique for recognizing attributes based on shape using data from 3D range sensors and targeting the same four types of attributes, we achieved a system that could support an environment with poor visibility and a situation in which the presence of multiple mobile devices may result in mutual shielding. We also showed that an autonomously moving mobility support device could recognize itself through information obtained by self-localization. Experimental results revealed that this system could achieve attribute recognition accuracy greater than $81 \%$ with an average of $90.2 \%$ for all four types of attributes even in a place with poor visibility and a situation in which the presence of multiple mobile devices may result in mutual shielding.

\section{Conclusion}

This paper described platform technologies for achieving a practical living support service in an ordinary living environ- ment. Targeting ordinary persons in addition to the elderly and persons in need of care giving, the idea behind such a service is to detect a person's intentions from brain activity, control home appliances and the ambient environment accordingly, convey the person's emotional state to others, etc. To reiterate, the target of these technologies is not only people in need of care giving or assistance. By "monitoring the brain," they are expected to enhance interpersonal communication in diverse scenarios and help create an environment for living a productive and fulfilling life as an individual. Our aim going forward is to provide a variety of practical services with these features in mind. Furthermore, in addition to the operation of various types of equipment by BMI, it will also be important to determine the user's current condition in more detail such as by identifying specific emotions. For example, learning about the user's emotional state should make it possible to provide the user with a comfortable environment automatically so that the user has no need to directly operate equipment. In future research, we will place importance on researching and developing technology that enables the environment to naturally support a person in the background without direct operations.

\section{Acknowledgement}

This research was supported by a contract with the Ministry of Internal Affairs and Communications entitled, 'Novel and innovative R\&D making use of brain structures'. A part of this research was funded by ImPACT Program of Council for Science, Technology and Innovation (Cabinet Office, Government of Japan). This research is a joint research by Keio University, Shimadzu Corporation, NTT Corporation, Sekisui House, Ltd. and Advanced Telecommunications Research Institute International.

\section{References}

[1] B. Blankertz, G. Dornhege, M. Krauledat, K.-R. Müller, and G. Curio, "The non-invasive Berlin brain-computer interface: Fast acquisition of effective performance in untrained subjects," NeuroImage, vol.37, no.2, pp.539-550, 2007.

[2] T. Carlson and J.D.R. Millán, "Brain-controlled wheelchairs: A robotic architecture," IEEE Robot. Automat. Mag., vol.20, no.1, pp.65-73, Jan. 2013.

[3] F. Aloise, F. Schettini, P. Aricó, F. Leotta, S. Salinari, D. Mattia, F. Babiloni, and F. Cincotti, "Toward domotic appliances control through a self-paced P300-based BCI," Proc. International Conference on Bio-inspired Systems and Signal Processing, pp.239-244, 2011.

[4] I. Iturrate, J.M. Antelis, A. Kubler, and J. Minguez, "A noninvasive brain-actuated wheelchair based on a P300 neurophysiological protocol and automated navigation," IEEE Trans. Robot., vol.25, no.3, pp.614-627, 2009.

[5] J.D. Millan, R. Rupp, G.R. Muller-Putz, R. Murray-Smith, G. Giuqlienmma, M. Tangermann, C. Vidaurre, F. Cincotti, A. Kubler, R. Leeb, C. Neuper, K.R. Muller, and D. Mattia, "Combining braincomputer interfaces and assistive technologies: State-of-the-art and challenges," Front Neurosci, vol.4, no.161, 2010.

[6] E. Aarts and B. de Ruyter, "New research perspectives on ambient intelligence," J. Ambient Intell. Smart Environ., vol.1, pp.5-14, 2009 
[7] D.J. Cook, "Multi-agent smart environments," J. Ambient Intell. Smart Environ., vol.1, pp.51-55, 2009.

[8] C.D. Kidd, R. Orr, G.D. Abowd, C.G. Atkeson, I.A. Essa, B. MacIntyre, E. Mynatt, T.E. Starner, and W. Newstetter, "The aware home: A living laboratory for ubiquitous computing research," Cooperative Buildings. Integrating Information, Organizations, and Architecture, Lecture Notes in Computer Science, vol.1670, pp.191198, 1999.

[9] T. Takeuchi, "Agent-based service platform for federating distributed heterogeneous sensors and its data processing," APAN Sensor Network WG, 2013.

[10] H. Morioka, A. Kanemura, S. Morimoto, T. Yoshioka, S. Oba, M. Kawanabe, and S. Ishii, "Decoding spatial attention by using cortical currents estimated from electroencephalography with near-infrared spectroscopy prior information," NeuroImage, vol.90, pp.128-139, 2014

[11] H. Morioka, A. Kanemura, J. Hirayama, M. Shikauchi, T. Ogawa, S. Ikeda, M. Kawanabe, and S. Ishii, "Learning a common dictionary for subject-transfer decoding with resting calibration," NeuroImage, vol.111, pp.167-178, 2015.

[12] A. Kanemura, Y. Morales, M. Kawanabe, H. Morioka, N. Kallakuri, T. Ikeda, T. Miyashita, N. Hagita, and S. Ishii, "A waypoint-based framework in brain-controlled smart home environments: Brain interfaces, domotics, and robotics integration," 2013 IEEE/RSJ International Conference on Intelligent Robots and Systems, pp.865-870, 2013

[13] A. Kanemura, Y. Morales, M. Kawanabe, H. Morioka, N. Kallakuri, T. Ikeda, T. Miyashita, N. Hagita, and S. Ishii, "A waypoint-based framework in brain-controlled smart home environments: Brain interfaces, domotics, and robotics integration," IEEE/RSJ International Conference on Intelligent Robots and Systems, pp.865-870, 2013.

[14] T. Ogawa, J. Hirayama, P. Gupta, H. Moriya, S. Yamaguchi, A. Ishikawa, Y. Inoue, M. Kawanabe, and S. Ishii, "Brain-machine interfaces for assistive smart homes: A feasibility study with wearable near-infrared spectroscopy," Proc. 2015 37th Annual International Conference of the IEEE Engineering in Medicine and Biology Society (EMBC), pp.1107-1110, 2015.

[15] L.Y. Morales-Saiki, T. Miyashita, and N. Hagita., "Human comfort for autonomous vehicles," Workshop "Assistance and service robotics in a human environment" in conjunction with IEEE/RSJ International Conference on Intelligent Robots and Systems, pp.27372743, 2014.

[16] C.-C. Chang and C.-J. Lin, "LIBSVM: A library for support vector machines," ACM Trans. Intell. Syst. Technol., vol.2, no.3, pp.1-27, 2011

[17] F. Scholkmann, S. Kleiser, A.J. Metz, R. Zimmermann, J.M. Pavia, U. Wolf, and M. Wolf, "A review on continuous wave functional near-infrared spectroscopy and imaging instrumentation and methodology," NeuroImage, vol.85, pp.6-27, 2014.

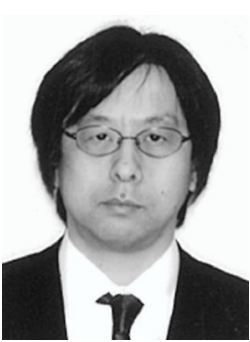

Takayuki Suyama is a Head of Dept. of Dynamic Brain Imaging, Cognitive Mechanisms Laboratories, Brain Information Communication Research Laboratory Group, Advanced Telecommunications Research Insutitute International (ATR). He received the B.E. and M.E. degrees in mechanical engineering from Osaka University in 1990 and 1992, respectively, and the Ph.D. degree in informatics from Kyoto University in 2007. He joined NTT Communication Science Laboratories in 1992 and studied a highlevel synthesis system for hardware. During 1999-2003, he worked for the Research and Development Center of NTT WEST Corporation. He returned to NTT Communication Science Laboratories in 2003. He joined ATR in 2014. His research interests are sensor network systems, distributed systems, ubiquitous computing and brain machine interface. He is a member of IEEE, ACM, IEICE and IPSJ. 